\title{
Die Restaurierung und ihre Werkstatt
}

Ein Rückblick auf das Jahr 2017

Die Bestandserhaltung umfasst ein weites Aufgabenspektrum, angefangen bei komplexen Restaurierungsmaßnahmen bis zu Aspekten der Konservierung, wie der sachgerechten Lagerung und Verpackung, Mengenentsäuerungen ganzer Bestandsgruppen, der Schimmelpilzbehandlung und Schädlingsbekämpfung, Digitalisierungsmaßnahmen, Schutzverfilmungen, der Exponatpflege bei Ausstellungen und dem Notfallmanagement bei Havarien.

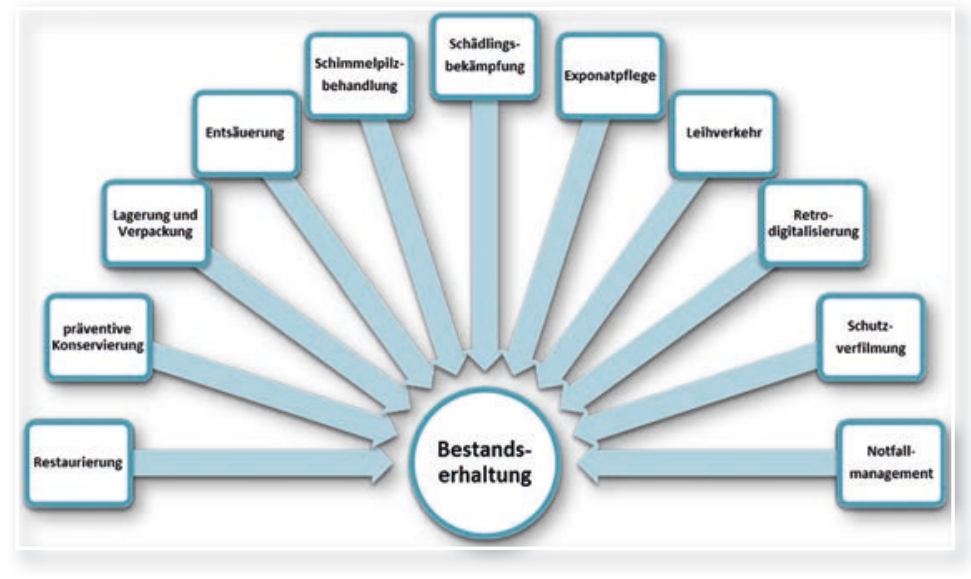

Abb. 1: Spektrum der Bestandserhaltung

Was genau passiert aber in der Restaurierungswerkstatt der WLB?

Hier werden Objekte, die durch Lagerung, Benutzung, Klimaeinwirkungen oder Alterungserscheinungen beschädigt worden sind, behandelt oder zur Außenvergabe koordiniert. Das Schadensbild reicht von losen Lagen und Rissen bzw. Fehlstellen am Papier über abgelöste Buchrücken oder abgeplatzte Teile des Lederbezugs bis zum Tinten- und Säurefraß. Die Restaurierung beschädigter Sammlungsobjekte gewährleistet, dass die historischen Sonderbestände auch weiterhin für die künftige Nutzung, für die Forschung oder für Ausstellungen zur Verfügung stehen. Insgesamt wurden im vergangenen Jahr rund 3.000 Objekte bearbeitet, sei es durch konservatorische Maßnahmen, restauratorische Behandlungen, als Vorbereitung für die Digitalisierung oder im Rahmen der Betreuung von Ausstellungen und des Leihverkehrs zwischen der WLB und anderen
Kulturinstitutionen. Die Dokumentation und Koordination dieser Vorgänge machen einen beträchtlichen Anteil dieser Arbeit aus.

\section{Interne und externe Restaurierungen}

Für die Historischen Sondersammlungen wurden 83 Einheiten im Haus restauratorisch behandelt und die Arbeiten protokolliert. Ein Chorbuch mit Tintenfraßschäden (Cod. mus. I fol. 32) und ein Stammbuch aus dem Jahr 1792 (Cod. hist. $8^{\circ} 341$ ) mussten aufwendig restauriert, dabei schriftlich und fotografisch dokumentiert werden.
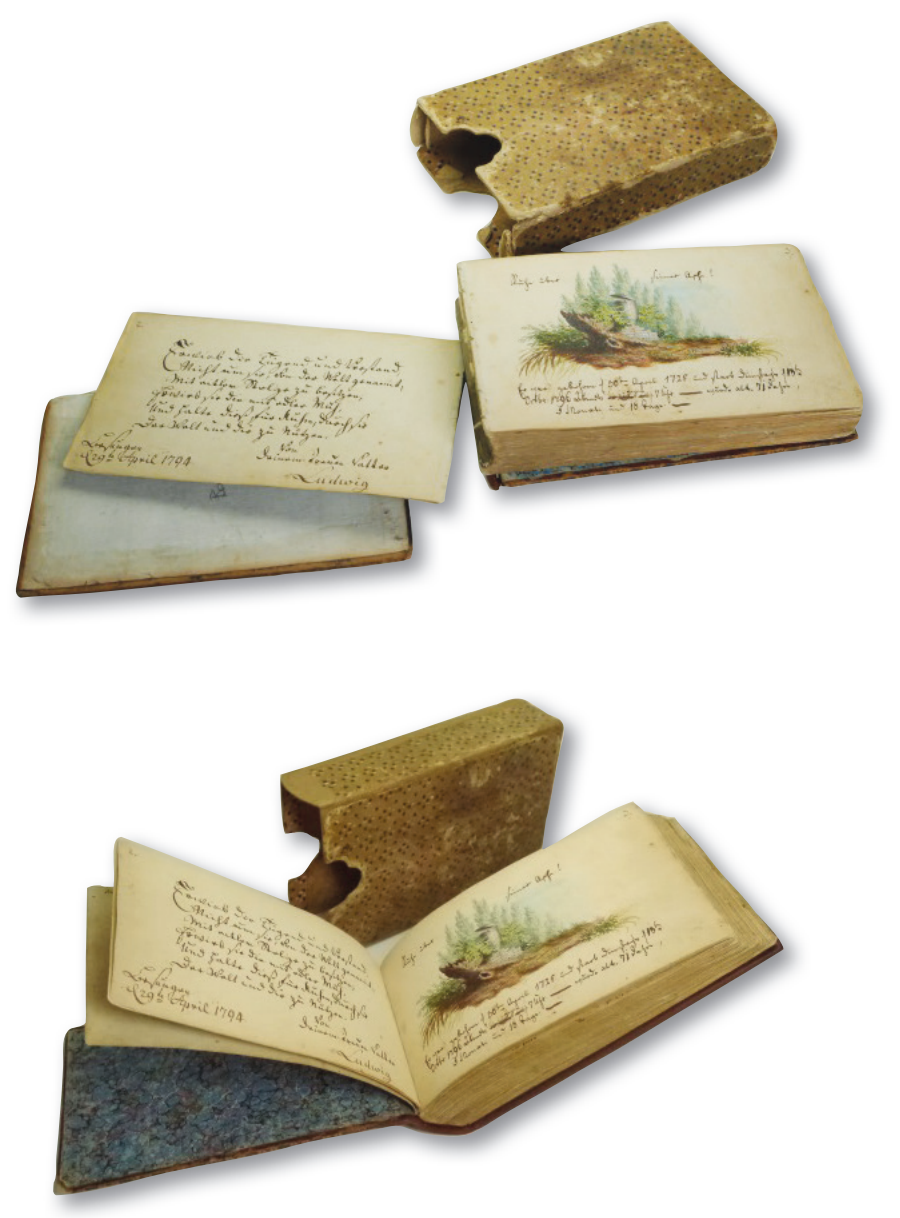

Abb. 2 und 3: Buch und Schuber (Cod.hist. $8^{\circ} 341$ ) vor und nach der Restaurierung

Von 75 nach außen vergebenen Restaurierungsaufträgen wurden die Arbeiten an 17 Bänden durch Buchpatenschaften finanziert. Dafür wurden die ausgesuchten Objekte der Sammlungen einge- 
hend geprüft, die Schäden und nötigen Maßnahmen genau beschrieben. Anschließend erfolgte die Vergabe der Bände zur Restaurierung an diverse Werkstätten. Für externe Restaurierungsaufträge müssen wiederholt Angebote spezialisierter Dienstleister angefordert oder verfahrenstechnische Ausschreibungen gemacht werden.

Eine langjährige Zusammenarbeit besteht mit dem Institut für Erhaltung (IfE) in Ludwigsburg. Im durch das IfE koordinierten Landesrestaurierungsprogramm konnten im Jahr 2017 zehn Handschriften und alte Drucke, 350 Fotografien der Bibliothek für Zeitgeschichte (BfZ) sowie zwölf Karten und Grafiken aufwendig restauriert werden. Zusätzliche Sachmittel ermöglichten es, mehrere Projekte durchzuführen: Hierzu gehört die Einbandrestaurierung von Bänden aus der Sammlung Nicolai, die in Kooperation mit der Werkstatt Johannes Schrempf (Esslingen) fortgeführt werden konnte.

Ebenfalls über das Ludwigsburger Institut für Erhaltung konnten 2.471 Einheiten aus der Sammlung der BfZ entsäuert werden; von Juli bis Dezember 2017 fertigten zwei Studentinnen von der Akademie der Bildenden Künste 256 Konservierungsbroschuren für diese entsäuerte Bestandsgruppe an.

Die Musikabteilung der WLB erhielt für 237 ihrer Einheiten aus Nachlässen neue, säurefreie und alterungsbeständige Verpackungen.

Besondere Erwähnung verdient die Modellprojektförderung der Koordinierungsstelle für den Erhalt des schriftlichen Kulturguts (KEK): Das Motto lautete im Jahr 2017 „Das besondere Format", wofür die Restaurierung von 13 großformatigen Wandzeitungen realisiert wurde.

\section{Konservierungsarbeiten und Bestandserhal- tungsprojekte}

Einen großen Anteil der Restaurierungsarbeiten betreffen die stets notwendigen konservatorischen Verpackungen und Bindearbeiten. 2017 konnten 778 Bände in 5 „Buchbindepartien“ bearbeitet und mit Schutzverpackungen versehen werden. Die Auftragssumme (ca. $35.000 €$ ) spiegelt die Dimension dieser Projekte wider, die meist unauffällig, als Routinen im Hintergrund erledigt und selten in ihrer konservatorischen Bedeutung wahrgenommen werden. Für diese Arbeiten sprechen sich die Handschriftenabteilung und die Abteilung der Alten Drucke eng mit der Restaurierung hinsichtlich
Auswahl und Vorbereitung der Partien für Schuber, Kassetten, Mappen und Bindearbeiten ab.

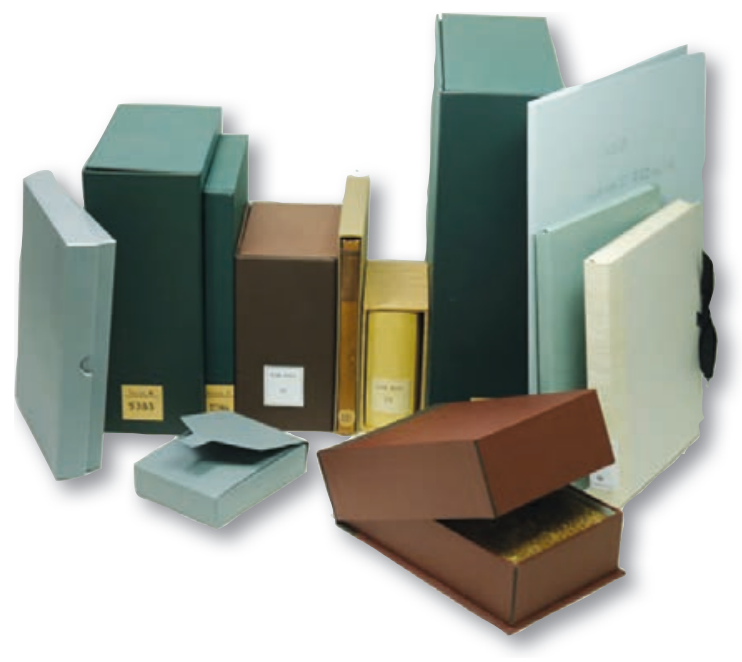

Abb. 4: Verpackungen als konservatorische Maßnahme

Für 390 weitere Einheiten wurden im Haus individuelle Verpackungen wie Mappen und Umschläge angefertigt. Hierfür wurden nach Vermessung der Objekte säurefreie Aktenumschläge zugeschnitten und gefalzt sowie individuelle Verpackungen und Kassetten angefertigt.

613 Bände aus dem Hauptlesesaal, Magazin und den Abteilungen Handschriften und Alte Drucke erhielten durch eine studentische Hilfskraft eine Trockenreinigung und individuell angefertigte Schutzumschläge.

Drei Grafiken aus den Grafischen Sammlungen wurden zu konservatorischen Zwecken entrahmt.

\section{Notfälle der Bestandserhaltung}

Aufgrund eines Wassereinbruchs im Magazin, der durch Bauarbeiten zum Jahresende 2016 entstanden war, musste die Trocknung des Wasserschadens an 446 Bände erfolgen. Ein Teil der Bände wurde direkt im Haus getrocknet, mehrere Bände konnten durch eine Gefriertrocknung gerettet werden, für einen weiteren Teil wurden Ersatzbeschaffungen geprüft.

\section{Ausstellungen und Leihgaben}

Bevor Objekte wie Bücher, Zeitschriften oder Karten in einer Ausstellung gezeigt werden können, ist in der Regel die konservatorische Expertise aus der Restaurierung gefragt. Dabei gilt es, nicht nur die klimatischen Bedingungen am Ausstellungsort zu prüfen, es müssen auch die Anforderungen für Verpackung, den Transport in Klimakisten und die 
Präsentation festgelegt werden. Die materialgerechte Exposition mit Buchstützen, das Fixieren einer Buchseite, das Montieren großformatiger Materialien, die Licht- und Temperaturverhältnisse, all das spielt eine Rolle, wenn ein Objekt das Haus für eine auswärtige Ausstellung verlässt. Hier sind abteilungsübergreifende Absprachen zwischen der jeweiligen Sammlungsleitung, der Restaurierung, dem Ausstellungswesen, Kunstspeditionen und der leihnehmenden Institution gefragt.

Im Jahr 2017 waren es 44 Leihgabenprotokolle für hochversicherte Objekte und insgesamt 150 externe Leihgaben, die in diesem Zusammenhang erstellt wurden. Dem ging die konservatorische Begutachtung der angefragten Leihgaben voraus. Vereinzelt mussten Exponate bis zur Vitrine am Zielort als Kurier begleitet werden, so in das Liebighaus nach Frankfurt, in das Erzbischöfliche Diözesanmuseum nach Paderborn, in das Museum der Universität Tübingen sowie in die Staatsgalerie Stuttgart.
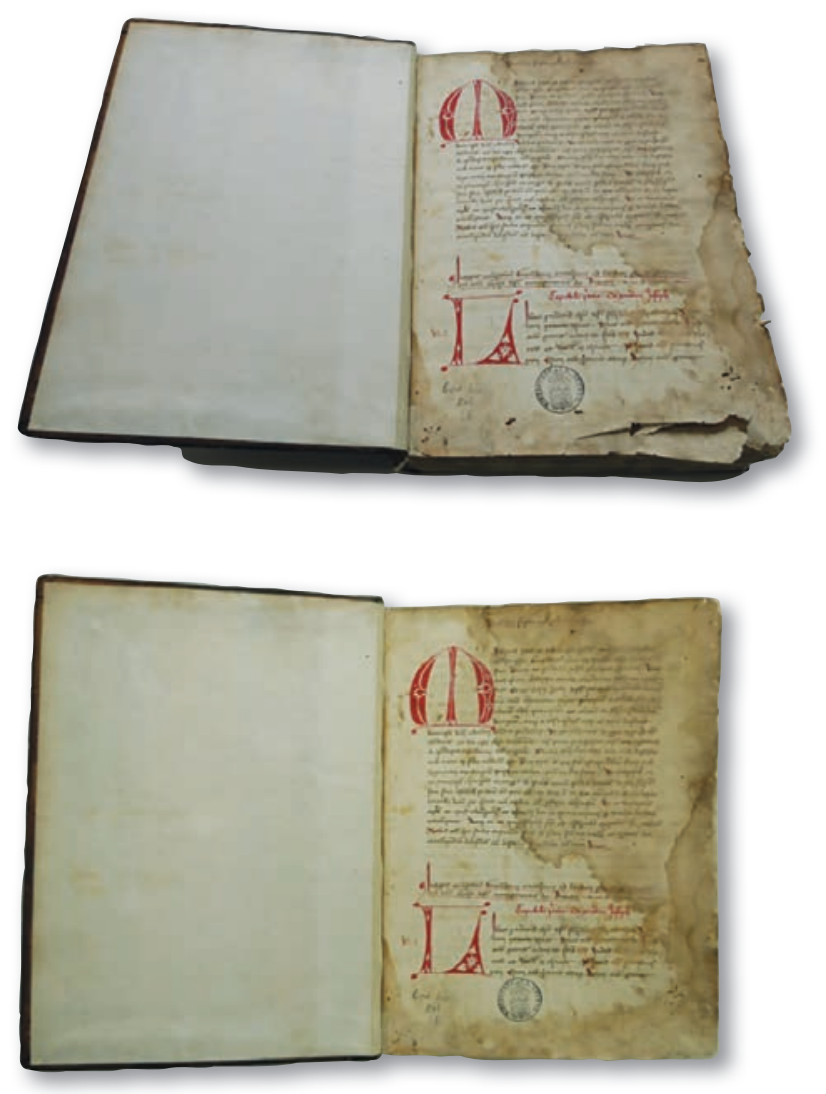

Abb. 5 und 6: Band vor und nach einer Sicherungsmaßnahme für eine Leihgabe

Vier Ausstellungen wurden umfassend durch vorbereitende Restaurierungsmaßnahmen, Präsentationshilfen, Leihgabenprotokolle, Verpacken und Auf- und Abbau unterstützt:
- „KEK und der »Neue Atlas für die Jugend « von Jakob Friedrich Klemm" - Präsentation der abgeschlossenen Modellprojektförderung 2016 durch die Koordinierungsstelle für die Erhaltung des schriftlichen Kulturguts (KEK), Württembergische Landesbibliothek

- „Objekt im Fokus: Untergang des Zarenreichs" - Präsentation in der Württembergischen Landesbibliothek

- "Le vent de la Réforme - Luther 1517 / Der Sturmwind der Reformation - Luther 1517“, in Kooperation mit der Bibliothèque nationale et universitaire de Strasbourg (BNU) in Straßburg

- „Freiheit - Wahrheit - Evangelium. Reformation in Württemberg", in Kooperation mit dem Staatsarchiv im Kunstgebäude Stuttgart

\section{Unterstützung der Digitalisierung}

Besonders die Nutzung fragiler und unikaler Objekte stellt für Bibliotheken häufig ein Dilemma dar. Eine bestandserhaltende Möglichkeit ist hierbei die Digitalisierung. Zuvor muss das Material durch die Restaurierung aus konservatorischer Perspektive gesichtet werden. Stellt sich ein Band beispielsweise als zu fragil heraus, müssen vor der Digitalisierung Restaurierungsmaßnahmen erfolgen, da sonst beim Aufschlagen oder Blättern weitere Schäden am Objekt drohen. Dieser Tätigkeitsbereich erfordert ebenfalls zahlreiche Absprachen mit den jeweiligen Sammlungsbereichen und der Digitalisierungsabteilung.

\section{Partnerschaften in der Ausbildung}

Studierende des Studiengangs Restaurierung der Staatlichen Akademie der Bildenden Künste durften im Rahmen ihrer Semesterarbeiten mehrere Bände aus dem Bestand der WLB bearbeiten. Zwei Praktikantinnen der Hochschule der Medien Stuttgart, ein externer Bibliotheksreferendar sowie vier Studierende der Bibliothekswissenschaft wurden in den "Grundlagen der Restaurierung und Bestandserhaltung in der WLB" unterrichtet. Sie erhielten eine Einführung in die Aufgaben und Abläufe der Restaurierungswerkstatt, Erläuterungen zu aktuellen Restaurierungsmaßnahmen und konservatorischen Aspekten. Mit kleinen Arbeitsproben wurden einzelne Themen vertieft. 

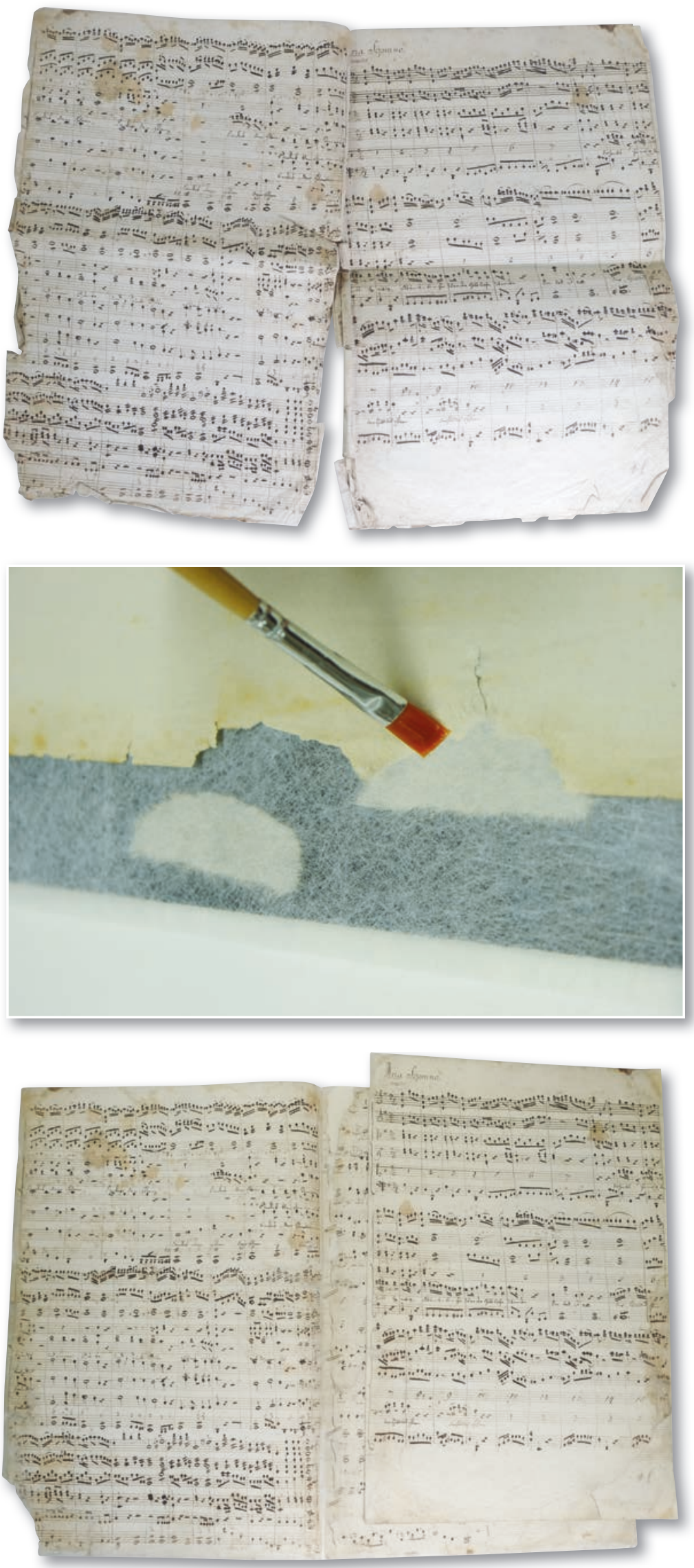

Abb. 7-9: Restaurierungsmaßnahmen im Vorfeld einer Digitalisierung

\section{Gremienarbeit und Fortbildung}

Restauratorinnen und Restauratoren besitzen die nötige Expertise, um Gefährdungen und Schäden zu erkennen, wissenschaftlich zu untersuchen und präzise zu dokumentieren sowie fachgerechte Empfehlungen für Konservierungs- und Restaurierungsmaßnahmen auszusprechen.

Die Aufgabe des Restaurierungsreferats ist das Management gefährdeten Kulturguts: Aufträge und Projekte müssen vorausschauend geplant und Drittmittel für den Originalerhalt eingeworben werden. In der Expertengruppe des Wissenschaftsministeriums fällt der Referentin für Restaurierung die Aufgabe zu, Anträge baden-württembergischer Bibliotheken auf Fördermittel für die Bestandserhaltung zu begutachten.

Die Restauratorin und die Referentin für Restaurierung der WLB sind in regionalen und nationalen Fachgruppen vernetzt, um beispielsweise bei Notfällen zu agieren sowie Aufträge schnell und passgenau an spezialisierte Werkstätten zu kanalisieren.

Aus dem Jahr 2017 sind neben der Gremienarbeit im Rahmen des Stuttgarter Notfallverbundes und den 23. Fachgesprächen des Arbeitskreises der NRW-Papierrestauratoren (Bonn, 3./4. April 2017) eine Fortbildung zu Bestandserhaltungsaspekten bei der Digitalisierung im Rahmen des Landesrestaurierungsprogrammes am Institut für Erhaltung zu nennen (Ludwigsburg, 4./5. Juli 2017).

\section{Umbau- und Modernisierungsmaßnahmen}

In den letzten Jahren fanden mehrere Umbaumaßnahmen innerhalb der Restaurierungswerkstatt statt. Es wurde eine neue Wässerungsanlage angebracht, die alten, fest installierten Tische mit Unterschränken aussortiert sowie ein defekter Gefahrenstoffschrank entsorgt. Die Modernisierung der Werkstatt geht mit der Neuanschaffung von Arbeitstischen mit einheitlicher Arbeitshöhe auf Rollen einher. Ein Tisch besitzt eine eingebaute Leuchtfläche, ein anderer ist höhenverstellbar. Der flexible Einsatz der Tischmodule ermöglicht die Anpassung an den individuellen Bedarf. Der neue Gefahrenstoffschrank hat eine Abluftfunktion durch einen aufgesetzten "In-Raum-Filter". Eine Analysestation für Untersuchungen ist mit Mikroskop, pH-Messgerät und mobilem Absaugsystem ausgestattet. Neu kommt auch ein Gefrierschrank für die Notfallvorsorge hinzu. 

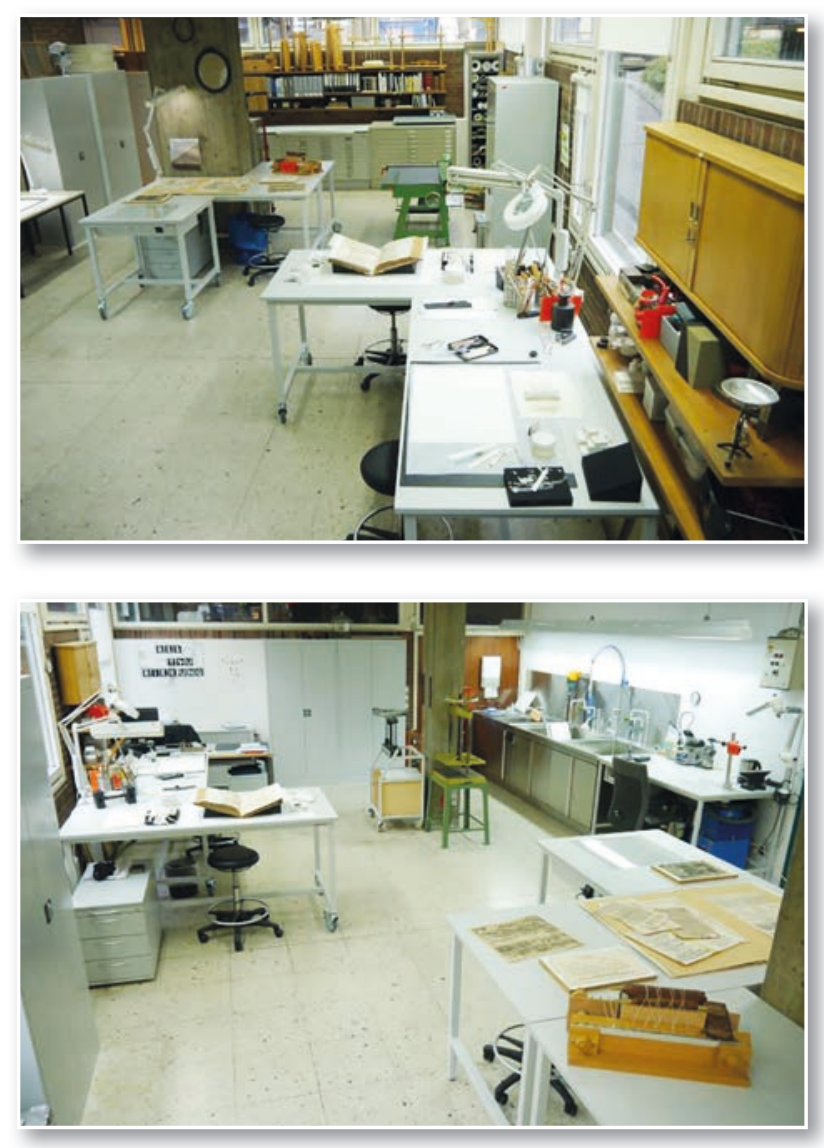

Abb. 10 und 11: Restaurierungswerkstatt nach der Modernisierung

\section{Öffentlichkeitsarbeit}

Interessierte lernen die Restaurierungswerkstatt der WLB im Rahmen einer Führung kennen und erfahren, was zu einer möglichst schonenden Lagerung und Benutzung des Bestandes gehört. Darüber hinaus führt Restauratorin Sonja Brandt verschiedene Arbeitsmethoden wie Trockenreinigung, Papierergänzung, wässrige Behandlungen sowie verschiedene Arbeitsmaterialien, konservatorische Verpackungen und Präsentationshilfen vor. Anlässe für Werkstattführungen in der Restaurierung stellten 2017 der Empfang der Buchpaten und eine Besuchergruppe aus dem Amt für Vermögen und Bau dar und sollen zukünftig erweitert werden. Eines der geplanten Projekte für 2018 stellt der Entwurf eines Werbeflyers für Bestandserhaltung und Restaurierung in der WLB dar. Damit soll der Öffentlichkeit auch ein Einblick in die Arbeiten der Bestandserhaltung, einem Kernbereich der Bibliothek, ermöglicht werden.

Ida Danciu / Sonja Brandt 